\title{
Spin-flip noise in a multiterminal spin valve
}

\author{
W. Belzig ${ }^{1}$ and M. Zareyan ${ }^{2,3}$ \\ ${ }^{1}$ Departement für Physik und Astronomie, Klingelbergstrasse 82, 4056 Basel, Switzerland \\ ${ }^{2}$ Max-Planck-Institut für Physik komplexer Systeme, Nöthnitzer Strasse 38, 01187 Dresden, Germany \\ ${ }^{3}$ Institute for Advanced Studies in Basic Sciences, 45195-159, Zanjan, Iran
}

(Received 2 March 2004; published 15 April 2004)

\begin{abstract}
We study shot noise and cross-correlations in a four terminal spin-valve geometry using a BoltzmannLangevin approach. The Fano factor (shot noise to current ratio) depends on the magnetic configuration of the leads and the spin-flip processes in the normal metal. In a four-terminal geometry, spin-flip processes are particularly prominent in the cross-correlations between terminals with opposite magnetization.
\end{abstract}

DOI: 10.1103/PhysRevB.69.140407

PACS number(s): 74.40.+k, 72.25.Rb, 73.23.-b

The discovery of the giant magnetoresistance effect in magnetic multilayers has boosted the interest in spindependent transport in recent years (for a review see, e.g., Ref. 1). Recently spin-dependent transport in metallic multiterminal structures has also been demonstrated experimentally. ${ }^{2}$ In combination with quantum transport effects the field is termed spintronics. ${ }^{3}$ One important aspect of quantum transport is the generation of shot noise in mesoscopic conductors, ${ }^{4,5}$ e.g., the suppression of the shot noise from its classical value due to Fermionic statistics. ${ }^{6-8}$

A particularly interesting phenomenon is the nonlocal correlation between currents in different terminals of a multiterminal structure. For a noninteracting fermionic system the cross-correlations are generally negative. ${ }^{9}$ In a one-channel beam splitter the negative sign was confirmed experimentally. ${ }^{10,11}$ If the electrons are injected from a superconductor, the cross-correlations may change sign and become positive. ${ }^{12}$ In these studies, however, the spin was only implicitly present due to the singlet pairing in the superconductor.

Current noise in ferromagnetic-normal metal structures, in which the spin degree of freedom plays an essential role, has so far attracted only little attention. In two-terminal spin valves it was shown that the noise depends on the relative magnetization angle in a different way than the conductance $^{13}$ and spin-flip scattering. ${ }^{14-16}$ Thus, the noise reveals additional information on the internal spin dynamics. Noise has been exploited to study the properties of localized spins $^{17}$ or probe quantum entanglement of itinerant spins. ${ }^{18}$

In this work we propose an instrument for the study of spin-dependent transport: the use of cross-correlations in a magnetic multiterminal structure. The basic idea is to use a four-terminal structure like that sketched in Fig. 1. An electron current flows from the left terminals to the right terminals and is passing a scattering region. In the absence of spin-flip scattering the currents of spin-up electrons and spindown electrons are independent, and the cross-correlations between different spin-currents vanish. However, spin-flip scattering can convert spin-up into spin-down electrons and vice versa. The resulting equilibration of the spin population leads to a weakened magnetoresistance effect. More importantly, however, current cross-correlations between the differently polarized terminals are induced by the spin flips and now contain additional information about the scattering region.
To this end we will study a four-terminal structure, in which the currents can be measured in all four terminals independently. The layout is shown in Fig. 1, in which the various currents are defined. For simplicity, we assume that all four terminals are coupled by tunnel junctions to one node. The node is assumed to have negligible resistance, but provides spin-flip scattering. The ferromagnetic character of the terminals is modeled by spin-dependent conductances of the tunnel junctions. The two left (right) terminals have chemical potential $V_{L}\left(V_{R}\right)$. In most of the final results we will assume zero temperature, but this is not crucial. Furthermore, we will assume fully polarized tunnel contacts, characterized by a conductance $g_{a \sigma}$, where $a=L, R$ denotes left and right terminals, and $\sigma=\uparrow, \downarrow$ stands for the spin directions (in equations we take $\uparrow=+1$ and $\downarrow=-1$ ).

The current fluctuations in our structure can be described in a Boltzmann-Langevin formalism. ${ }^{19}$ The time-dependent spin-polarized currents at energy $E$ through contact $a \sigma$ are written as

$$
I_{a \sigma}(t, E)=g_{a \sigma}\left[f_{a \sigma}(E)-f_{c \sigma}(E)-\delta f_{c \sigma}(t, E)\right]+\delta I_{a \sigma}(t, E) .
$$

The averaged occupations of the terminals are denoted by $f_{a \sigma}(E)$, the one of the central node by $f_{c \sigma}(E)$. The occupa- (a)

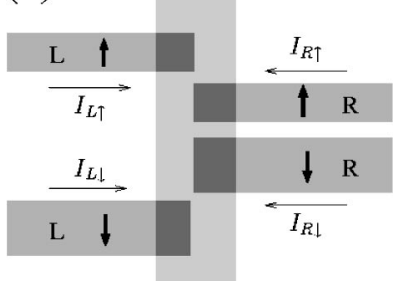

(b)

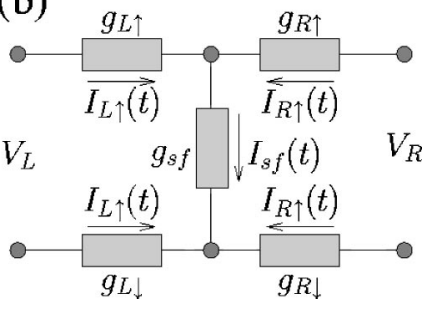

FIG. 1. Four-terminal setup to measure spin-flip correlations. (a) A possible experimental realization with a normal diffusive metal strip, on which four ferromagnetic strips are deposited (of different widths to facilitate different magnetization orientations). The total length of the diffusive metal underneath the ferromagnetic contacts should be less than the spin-diffusion length in the normal metal. (b) A theoretical model of the device. The spin $\uparrow(\downarrow)$ current is flowing in the upper (lower) branch. Spin-flip scattering connects the two spin branches and is modeled as a resistor which also induces additional fluctuation. 
tion of the central node is fluctuating as $\delta f_{c \sigma}(t, E)$. The Langevin source $\delta I_{a \sigma}(t, E)$ induces fluctuations due to the probabilistic scattering in contact $a \sigma$. We assume elastic transport in the following, so all equations are understood to be at the same energy $E$. Since we assume tunnel contacts, the fluctuations are Poissonian and given by ${ }^{4}$

$$
\begin{aligned}
\left\langle\delta I_{a \sigma}(t) \delta I_{a^{\prime} \sigma^{\prime}}\left(t^{\prime}\right)\right\rangle= & g_{a \sigma} \delta_{\sigma \sigma^{\prime}} \delta_{a a^{\prime}} \delta\left(t-t^{\prime}\right) \\
& \times\left[f_{a \sigma}+f_{c \sigma}-2 f_{a \sigma} f_{c \sigma}\right] .
\end{aligned}
$$

The brackets $\langle\cdots\rangle$ denote averaging over the fluctuations. The conservation of the total current at all times $t$ leads to the conservation law ${ }^{20}$

$$
\sum_{a, \sigma} I_{a \sigma}(t)=0
$$

The equation presented so far describes the transport of two unconnected circuits for spin-up and spin-down electrons, i.e., the spin current is conserved in addition to the total current. Spin-flip scattering on the dot leads to a nonconserved spin current, which we write as

$$
\sum_{a, \sigma} \sigma I_{a \sigma}(t)=2 g_{s f}\left[f_{c \uparrow}+\delta f_{c \uparrow}(t)-f_{c \downarrow}-\delta f_{c \downarrow}(t)\right]+2 \delta I_{s f}(t) .
$$

Here we introduced a phenomenological spin-flip conductance $g_{s f}$, which connects the two spin occupations on the node. ${ }^{21}$ Correspondingly, we added an additional Langevin source $\delta I_{s f}(t)$, which is related to the probabilistic spin scattering and has a correlation function ${ }^{22}$

$$
\left\langle\delta I_{s f}(t) \delta I_{s f}\left(t^{\prime}\right)\right\rangle=g_{s f} \delta\left(t-t^{\prime}\right)\left[f_{c \uparrow}\left(1-f_{c \downarrow}\right)+f_{c \downarrow}\left(1-f_{c \uparrow}\right)\right] .
$$

Equations (1)-(5) form a complete set and determine the average currents and the current noise of our system. Solving for the average occupations of the node we obtain

$$
f_{c \sigma}=\left[\left(g_{-\sigma} g_{L \sigma}+g_{s f} g_{L}\right) f_{L}+\left(g_{-\sigma} g_{R \sigma}+g_{s f} g_{R}\right) f_{R}\right] / Z .
$$

Here we introduced $g_{\sigma}=g_{L \sigma}+g_{R \sigma}, g_{L(R)}=g_{L(R) \uparrow}+g_{L(R) \downarrow}$, and $Z=g_{\uparrow} g_{\downarrow}+\left(g_{\uparrow}+g_{\downarrow}\right) g_{s f}$. The average currents are then

$$
I_{L \sigma}=\frac{g_{L \sigma}}{Z}\left[g_{R \sigma} g_{-\sigma}+g_{R} g_{s f}\right]\left(f_{L}-f_{R}\right),
$$

and the currents through the right terminals are obtained by interchanging $R \leftrightarrow L$ in Eq. (7). The fluctuating occupations on the node are

$$
\begin{aligned}
\delta f_{c \sigma}(t)= & {\left[\left(g_{-\sigma}+g_{s f}\right) \delta I_{\sigma}(t)+g_{s f} \delta I_{-\sigma}(t)\right.} \\
& \left.+g_{-\sigma} \sigma \delta I_{s f}(t)\right] / Z,
\end{aligned}
$$

where we introduced $\delta I_{\sigma}(t)=\delta I_{1 \sigma}(t)+\delta I_{-1 \sigma}(t)$. The total fluctuations of the current in a terminal are obtained from $\Delta I_{a \sigma}(t)=\delta I_{a \sigma}(t)-g_{a \sigma} \delta f_{c \sigma}(t)$ and we find

$$
\begin{aligned}
\Delta I_{L \sigma}= & \frac{1}{Z}\left[\left(g_{R \sigma} g_{-\sigma}+\left(g_{-\sigma}+g_{R \sigma}\right) g_{s f}\right) \delta I_{L \sigma}\right. \\
& -g_{L \sigma}\left(g_{-\sigma}+g_{s f}\right) \delta I_{R \sigma}+\sigma g_{L \sigma} g_{-\sigma} \delta I_{s f}-g_{L \sigma} g_{s f} \\
& \left.\times\left(\delta I_{L-\sigma}+\delta I_{R-\sigma}\right)\right] .
\end{aligned}
$$

Now we can calculate all possible current correlators in the left terminals, defined by

$$
S_{L \sigma \sigma^{\prime}}=\int_{-\infty}^{\infty} d \tau\left\langle\Delta I_{L \sigma}(t+\tau) \Delta I_{L \sigma^{\prime}}(t)\right\rangle .
$$

The total current noise in the left terminals is

$$
S_{L}=S_{L \uparrow \uparrow}+S_{L \downarrow \downarrow}+2 S_{L \uparrow \downarrow}
$$

Of course the same quantities can be calculated for the right terminals. From particle conservation it follows that $S_{L}$ $=S_{R}$, but in the presence of spin-flip scattering the individual correlators can differ. For convenience we also define a Fano factor $F=S_{L} / e|I|$, where $I=I_{L \uparrow}+I_{L \downarrow}$ is the total current.

Let us now turn to the four-terminal structure, displayed in Fig. 1(b), and study the effect of spin-flip scattering on the current noise and cross-correlation. We will restrict ourselves to zero temperature from now on. Assuming a bias voltage $V$ is applied between the right and the left terminals, the occupations are $f_{L}=1$ and $f_{R}=0$ in the energy range $0 \leqslant E$ $\leqslant e V$. We will in particular focus on cross-correlations between terminals with opposite magnetization directions. For the cross-correlations at the left side we find

$$
\begin{aligned}
S_{L \uparrow \downarrow}= & -g_{s f}|e V| \frac{g_{L \uparrow} g_{L \downarrow}}{Z^{3}} \sum_{\sigma=\uparrow \downarrow}\left\{\left[g_{-\sigma} g_{R \sigma}+\left(g_{-\sigma}+g_{R \sigma}\right) g_{s f}\right]\right. \\
& \times\left(g_{s f} g_{R}+g_{-\sigma} g_{R \sigma}\right) \\
& -g_{R \sigma}\left(g_{-\sigma}+g_{s f}\right)\left(g_{-\sigma} g_{L \sigma}+g_{s f} g_{L}\right) \\
& \left.+\frac{g_{\downarrow} g_{\uparrow}}{Z}\left(g_{-\sigma} g_{L \sigma}+g_{s f} g_{L}\right)\left(g_{\sigma} g_{R-\sigma}+g_{s f} g_{R}\right)\right\} . \quad(12)
\end{aligned}
$$

It can be shown, that the cross-correlations are always negative, as it should be. ${ }^{9}$ The full current noise can be written as

$$
\begin{aligned}
S_{L}= & \frac{|e V|}{Z^{3}} \sum_{\sigma=\uparrow \downarrow}\left[g_{L \sigma}\left(g_{s f} g_{R}+g_{-\sigma} g_{R \sigma}\right)^{3}+g_{R \sigma}\left(g_{s f} g_{L}\right.\right. \\
& \left.+g_{-\sigma} g_{L \sigma}\right)^{3}+\frac{g_{s f}}{Z}\left(g_{\downarrow} g_{L \uparrow}-g_{\uparrow} g_{L \downarrow}\right)^{2}\left(g_{s f} g_{R}+g_{\sigma} g_{R-\sigma}\right) \\
& \left.\times\left(g_{s f} g_{L}+g_{-\sigma} g_{L \sigma}\right)\right] .
\end{aligned}
$$

This generalizes the result of Refs. 14 and 15 to arbitrary polarization, since the full noise (13) is the same as for a two-terminal contact.

We now discuss analytical results in several simple cases. In lowest order in $g_{s f} /\left(g_{\uparrow}+g_{\downarrow}\right)$ the zero-frequency crosscorrelations (12) between the currents in the left terminals reduce to 


$$
\frac{S_{L \uparrow \downarrow}}{|e V|}=-2 g_{s f} \frac{g_{L \uparrow} g_{L \downarrow}}{g_{\uparrow}^{2} g_{\downarrow}^{2}}\left[g_{R \uparrow} g_{R \downarrow}+\frac{\left(g_{L \downarrow} g_{R \uparrow}-g_{L \uparrow} g_{R \downarrow}\right)^{2}}{g_{\uparrow} g_{\downarrow}}\right] .
$$

The first term is also present in a spin-symmetric situation, and is caused by the additional current path opened by the spin-flip scattering. The second term in Eq. (14) depends on the amount of spin accumulation on the central metal, i.e., it is proportional to $\left(f_{c \uparrow}-f_{c \downarrow}\right)^{2}$.

Now let us consider the symmetric "ferromagnetic" configuration $g_{L \uparrow}=g_{R \uparrow}=g_{\uparrow} / 2$ and $g_{L \downarrow}=g_{R \downarrow}=g_{\downarrow} / 2$. Note that also $g_{L}=g_{R}$ follows in this configuration. The crosscorrelations in the "ferromagnetic" configuration are

$$
S_{L \uparrow \downarrow}=-\frac{g_{s f}}{8} \frac{g_{\uparrow} g_{\downarrow}}{g_{\uparrow} g_{\downarrow}+g_{s f}\left(g_{\uparrow}+g_{\downarrow}\right)}|e V| .
$$

Thus, in the limit of strong spin-flip scattering the crosscorrelations become independent on $g_{s f}$. Next we consider the symmetric "antiferromagnetic" configuration $g_{L \uparrow}=g_{R \downarrow}$ $=g_{1}$ and $g_{L \downarrow}=g_{R \uparrow}=g_{2}$. For the cross-correlations we obtain

$$
\begin{aligned}
\frac{S_{L \uparrow \downarrow}}{|e V|}= & -\frac{g_{s f} g_{1} g_{2}}{2 g^{2}\left(g+2 g_{s f}\right)^{4}}\left[g\left(g+2 g_{s f}\right)^{3}+\left(g_{1}-g_{2}\right)^{2}\right. \\
& \left.\times\left(3 g^{2}+6 g g_{s f}+4 g_{s f^{2}}\right)\right],
\end{aligned}
$$

where we introduced the abbreviation $g=g_{1}+g_{2}$. Again, the second term in the brackets in Eq. (16) is proportional to the spin accumulation of the island, which enhances the spin-flip induced cross-correlations.

It is also interesting to study the shot noise of the total current in our setup. Evidently, the corresponding Fano factor is equivalent to a two-terminal structure with arbitrarily polarized contacts. In the simplified case of a two-terminal geometry with fully polarized contacts two different configurations are possible. Either both terminals have the same spin direction or the opposite configuration. In the first case we can take $g_{\downarrow}=0$. There is no effect of the spin-flip scattering and we obtain for the Fano factor $F=\left(g_{L}^{2}+g_{R}^{2}\right) /\left(g_{L}+g_{R}\right)^{2}$, in agreement with the known results. ${ }^{4}$ If the two terminals have different spin orientations ("antiferromagnetic" configuration), the situation is completely different, since transport is allowed only by spin-flip scattering. We take $g_{L \downarrow}$ $=g_{R \uparrow}=0$. The Fano factor is

$$
F=1-2 g_{s f} g_{L} g_{R} \frac{\left(g_{L}+g_{R}\right)\left(g_{L}+g_{s f}\right)\left(g_{R}+g_{s f}\right)}{\left(g_{L} g_{R}+\left(g_{L}+g_{R}\right) g_{s f}\right)^{3}},
$$

where we have used the result for the mean current $I$ $=g_{s f} g_{L} g_{R} /\left[g_{L} g_{R}+\left(g_{L}+g_{R}\right) g_{s f}\right]$. The Fano factor given in Eq. (17) interpolates between the Poisson limit $F=1$ for $g_{s f} \ll g_{L}+g_{R}$ and the result for the double barrier junction $F=\left(g_{L}^{2}+g_{R}^{2}\right) /\left(g_{L}+g_{R}\right)^{2}$ for $g_{s f} \gg g_{L}+g_{R}$, coinciding with two-terminal "ferromagnetic" configuration. ${ }^{15}$ For the symmetric ferromagnetic configuration the Fano factor of the full current noise is $1 / 2$ for arbitrary polarizations, i.e., we recover the usual suppression of the shot noise, characteristic

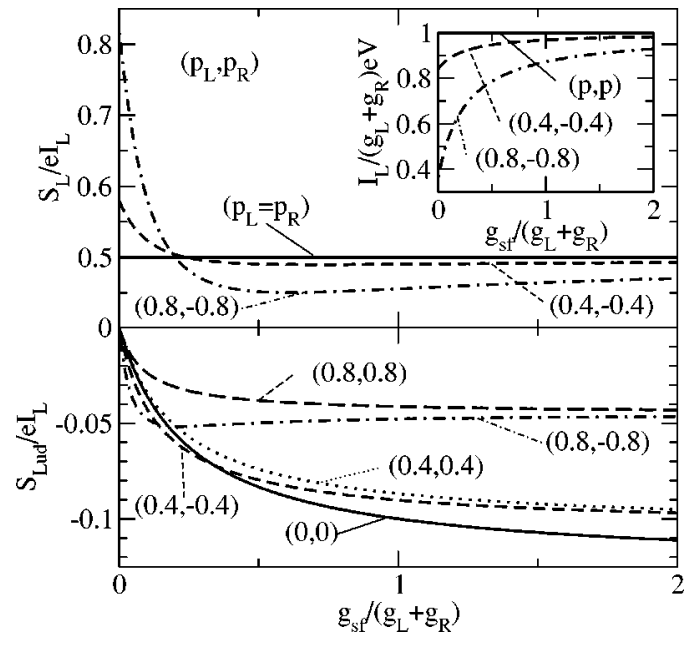

FIG. 2. Cross-correlations, Fano factor, and average currents (symmetric case). We assume symmetric contacts $g_{L}=g_{R}$ and parametrize the magnetic properties with the spin polarization $p_{L(R)}$ $=\left(g_{L(R) \uparrow}-g_{L(R) \downarrow}\right) /\left(g_{L(R) \uparrow}+g_{L(R) \downarrow}\right)$. The upper part shows the Fano factor of the current fluctuations in the left contacts for different polarization configurations. Inset: average current. The lower part shows the spin-flip-induced cross-correlations between $\uparrow$ and $\downarrow$ currents in the left terminals.

for a symmetric double barrier structure. On the other hand, the Fano factor for the symmetric antiferromagnetic configuration is

$$
F=\frac{1}{2}\left[1-\frac{\left(g_{1}-g_{2}\right)^{2}}{\left(g+2 g_{s f}\right)^{2}}\left(\frac{2 g_{s f}^{2}}{g g_{s f}+2 g_{1} g_{2}}-\frac{g}{g+2 g_{s f}}\right)\right] .
$$

The second term in the square brackets in Eq. (18) can be either positive or negative. In the latter case $F$ drops below the symmetric double barrier value of $1 / 2$.

The transport properties for symmetric junctions are summarized in Fig. 2. For equal polarizations of both sides there is no effect of spin-flip scattering on the Fano factor and average currents. However, the cross-correlations do depend on the polarizations even in this case. For small $g_{s f}$ the cross-correlations rapidly increase in magnitude. For $g_{s f}$ $\gg g_{L}+g_{R}$ the cross-correlations become independent of the relative polarizations. Their absolute value, however, depends strongly on the absolute value of the polarization. For antiparallel polarizations the Fano factor differs strongly from its value $1 / 2$ in the unpolarized case, see Eq. (18). With an increasing spin-flip scattering rate, the Fano factor goes from a value larger than $1 / 2$ through a minimum, which is always lower that $1 / 2$.

Let us now turn to the general case of asymmetric junctions. The noise correlations are plotted in Fig. 3 for $g_{L}$ $=4 g_{R}$ and various configurations of the polarizations 0.3 and 0.7. The cross-correlations, in particular for weak spin-flip scattering, differ now drastically for the different configurations. In particular, the cross-correlations in the antiferromagnetic configurations are strongly enhanced as a result of the larger spin accumulation in comparison to the ferromagnetic configuration. The Fano factors and the average cur- 


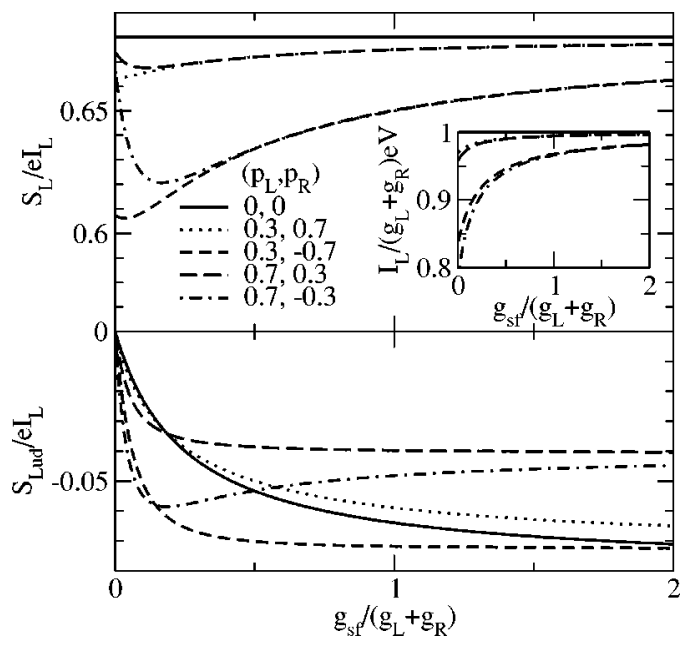

FIG. 3. Cross-correlations, Fano factor, and average currents (asymmetric case). We take here $g_{L}=4 g_{R}$. The definition of the polarizations are taken over from Fig. 2.

rents are also different for all parameter combinations. However, the variations of the Fano factors are small, i.e., they are always close to the unpolarized case.

Finally, we would like to comment on the experimental realization. For the determination of the cross-correlations it is crucial that the two spin currents are extracted at different terminals. The injection can also be done with one terminal, which can even be unpolarized. A more flexible fourterminal design is favorable, since different polarization configurations can then be obtained by exchanging the potentials at the different terminals. No change of the magnetization is necessary in that case. A structure like the one we have proposed in the left panel of Fig. 1 has recently been realized experimentally, ${ }^{23}$ although noise correlations have not been measured yet.

In conclusion we have suggested using shot noise and cross-correlations as a tool to study magnetotransport in mesoscopic spin valves. Measuring cross-correlations between currents in terminals with opposite spin orientations gives direct access to the spin-flip scattering rate. In the present work we have assumed fully polarized terminals, but a generalization to arbitrary polarizations is straightforward.

Note added. After submission of this paper a related work appeared, in which a similar model was studied (Ref. 24).

We acknowledge discussions with C. Bruder. W. B. was financially supported by the Swiss NSF and the NCCR Nanoscience. M. Z. thanks the University of Basel for hospitality.
${ }^{1}$ M.A.M. Gijs and G.E.W. Bauer, Adv. Phys. 46, 285 (1997).

${ }^{2}$ F.J. Jedema et al., Nature (London) 410, 345 (2000); 416, 713 (2002).

${ }^{3}$ Semiconductor Spintronics and Quantum Computation, edited by D. D. Awschalom, D. Loss, and N. Samarth (Springer, Berlin, 2002).

${ }^{4}$ Ya.M. Blanter and M. Büttiker, Phys. Rep. 336, 1 (2000).

${ }^{5}$ Quantum Noise in Mesoscopic Physics, edited by Yu. V. Nazarov (Kluwer, Dordrecht, 2003).

${ }^{6}$ V.A. Khlus, Sov. Phys. JETP 66, 1243 (1987).

${ }^{7}$ G.B. Lesovik, JETP Lett. 49, 592 (1989).

${ }^{8}$ M. Büttiker, Phys. Rev. Lett. 65, 2901 (1990).

${ }^{9}$ M. Büttiker, Phys. Rev. B 46, 12485 (1992).

${ }^{10}$ M. Henny et al., Science 284, 296 (1999); S. Oberholzer et al., Physica E 6, 314 (2000).

${ }^{11}$ W.D. Oliver et al., Science 284, 299 (1999).

${ }^{12}$ T. Martin, Phys. Lett. A 220, 137 (1996); M.P. Anantram and S. Datta, Phys. Rev. B 53, 16390 (1996); G.B. Lesovik et al., ibid. 60, 11935 (1999); J. Torres and T. Martin, Eur. Phys. J. B 12, 319 (1999); J. Börlin et al., Phys. Rev. Lett. 88, 197001 (2002); P. Samuelsson and M. Büttiker, ibid. 89, 046601 (2002); F. Taddei and R. Fazio, Phys. Rev. B 65, 134522 (2002); M. Shechter, Y. Imry, and Y. Levinson, ibid. 64, 224513 (2001).
${ }^{13}$ Y. Tserkovnyak and A. Brataas, Phys. Rev. B 64, 214402 (2001).

${ }^{14}$ A. Lamacraft, Phys. Rev. B 69, 081301(R) (2004).

${ }^{15}$ E.G. Mishchenko, Phys. Rev. B 68, 100409 (2003).

${ }^{16}$ E.G. Mishchenko, A. Brataas, and Y. Tserkovnyak, Phys. Rev. B 69, 073305 (2004).

${ }^{17}$ H.-A. Engel and D. Loss, Phys. Rev. B 65, 195321 (2002).

${ }^{18}$ D. Loss and E.V. Sukhorukov, Phys. Rev. Lett. 84, 1035 (2000); G. Burkard et al., Phys. Rev. B 61, R16303 (2000); J.C. Egues et al., Phys. Rev. Lett. 89, 176401 (2002).

${ }^{19}$ K.E. Nagaev, Phys. Lett. A 169, 103 (1992); Phys. Rev. B 57, 4628 (1998).

${ }^{20}$ We neglect all charging effects, i.e., we assume that $g_{a \sigma} \gg e^{2} / h$. We are also only interested here in current fluctuations on time scales longer than all $R C$ times.

${ }^{21}$ A. Brataas, Yu.V. Nazarov, J. Inoue, and G.E.W. Bauer, Phys. Rev. B 59, 93 (1999).

${ }^{22}$ The same correlation function was independently derived in Ref. 15 .

${ }^{23}$ M. Zaffalon and B.J. van Wees, Phys. Rev. Lett. 91, 186601 (2003).

${ }^{24}$ D. Sanchéz, R. Lopéz, P. Samuelsson, and M. Büttiker, Phys. Rev. B 68, 214501 (2003). 